\title{
LA RUGOSCOPIA PALATINA FORENSE COMO METODO DE IDENTIFICACION HUMANA A TRAVES DEL ANALISIS COMPARATIVO
}

\author{
Rugoscopia palatina as the Forensic Identification \\ Method of Human through the comparative analysis
}

* Gustavo Antonio Ramos Matute

\section{RESUMEN}

Introducción. La Identificación es el método o sistema empleado para fijar de la manera más segura la identidad de una persona en la vida jurídica, tanto civil como penal y evitar confundirla con otra. La Rugoscopia es una técnica de identificación odonto-estomatológica que se preocupa por estudiar, clasificar y registrar las rugosidades palatinas. Objetivo. Proporcionar elementos de juicio para la identificación de personas vivas o muertas a través de estructuras anatómicas específicas dentro de la cavidad oral. Pacientes y Metódos. Para la recolección de datos se realizó la toma de impresión del maxilar, en veinte pacientes atendidos en la clínica de la Carrera de Odontología de la Universidad Nacional Autonoma de Honduras en el Valle de Sula (UNAH-VS), luego se procedió a la Calcorugoscopia. El duplicado obtenido se dividió por la línea media y se registraron las formas y números de formas palatinas de cada lado. El paso siguiente fue el llenado de ficha de cada uno de los modelos pertenecientes a los individuos. Resultados. De los 20 pacientes estudiados la forma más común encontrada en el paladar derecho es las curva (24 rugas), en el paladar izquierdo fue la recta (27 rugas). Conclusiones. Las

*Odontologo Forense. Profesor de la Escuela Universitaria de Ciencias de la Salud, Universidad Nacional Autonoma de Honduras en el Valle de Sula ( EUCS-UNAHVS )

Dirigir correspondencia a: gramosmatute@gmail.com

Recibido: 3 de Marzo 2,015 Aprobado 15 de Julio 2,015
Rugas Palatinas son diferentes de un individuo a otro, en una misma persona ambas mitades del paladar son diferentes. La Calcorusgoscopia es un método de identificación que no permite la presunción.

\section{PALABRAS CLAVE}

Identificación, odontologia forense, paladar.

\section{ABSTRACT}

Introduction. The identification is the method or system used to fix the safest way the identity of a person in both civil and criminal legal life and avoid confusing it with another. The Rugoscopia is an identification technique Odonto-Stomatology who cares about studying, classifying and recording the palatine ridges. Objective. Provide evidence for the identification of persons living or dead through specific anatomical structures within the oral cavity. Patients and Methods. The data collection of impression the maxilla, in twenty patients arrived at the clinic Dentistry race was held. Then he proceeded to Calcorugoscopia. The duplicate obtained was divided by the midline and shapes and forms palatal numbers were recorded on each side. The next step was filling record of each of the models belonging to the individuos. Results Studies showed the form that occurs most frequently is the curve (24), the least presented is angled and it never showed it in a circle, different ways from 
one side of the palate and another, most often on the right side the left side Conclusions. The palatine rugas differ from one individual to another, within the same person both halves of the palate are different also in the same person roughness on the right side are different from the left side and is an identification method that does not allow the presumption.

\section{KEYWORD}

Identification, forensic dentistry, palate.

\section{INTRODUCCION}

Cada individuo se distingue de otros por un conjunto de signos externos que permiten identificarlos. Reconocer un cadáver es buscar los signos físicos mediante los cuales es posible identificar la personalidad civil de un desaparecido; tiene como finalidad establecer la realidad del fallecimiento.

Los métodos de identificación de un cuerpo desconocido incluyen características dentarias y huellas dactilares. ${ }^{(1)}$ La Dactiloscopia es una ciencia que estudia los relieves y crestas que se forman en las yemas de los dedos los cuales nos permiten tener nuestra identidad siendo única en cada ser humano.(2) Así como la Grafología es un test proyectivo que permite obtener un conocimiento profundo de la personalidad del individuo ya que relaciona directamente la escritura, con la psicología humana; en la particularidad de los rasgos se percibe la individualidad del escribiente. ${ }^{(3)}$

Las presunciones son una prueba indirecta que consiste en deducir, partiendo de un hecho base, un hecho consecuencia. Por ello, es definida, en términos generales, como la averiguación de un hecho desconocido, deduciéndolo de otro conocido, ${ }^{(4)}$ estas no aplican en campos tan delicados y que requieren conocimiento técnico especializado o científico en la materia, como lo es el caso de reconocimiento odontológico forense.

A este respecto podemos decir que toda metodología de investigación de un cuerpo o varios cuerpos debe de llevar a dictámenes cuyos resultados sean precisos, lógicos e imparciales. Este tipo de procedimiento es objetivo, ya que en ningún punto se traslapan es decir en el caso de la ficha antemorten y posmorten realizadas por personas y grupos distintos. ${ }^{(5,6,7)}$

La rugoscopia forense es una técnica de identificación que estudia, clasifica y registra las rugosidades palatinas con el propósito de ser utilizados en casos complejos de identificación en que no se pueda o sea posible la utilización de los métodos convencionales como la queiloscopia que es un método de identificación odontológica usada en la Criminalística, basada en el estudio, registro y clasificación de los surcos presentes en la mucosa labial. ${ }^{(8,9)}$

Las rugas palatinas son propias e inalterables permitiendo cotejar un registro ante mortem para establecer la identificación en cadáveres sometidos a procesos de destrucción generalizada.

Como elemento de identificación las rugas palatinas poseen características que permiten utilizarlas: 
- Unicidad: Apenas un individuo puede tenerlas.

- Inmutabilidad: No cambia nunca de forma,no pueden cambiar de posición y duran toda la vida; no son susceptibles de perderse por el contacto con la superficie o acción compresiva de prótesis dentales totales o removibles.

- Individualidad: Son absolutamente diferentes de una persona a otra.

- Perenne: No cambian desde el nacimiento hasta la muerte.

En el rugograma se describen y registran las rugas palatinas de acuerdo a su forma, posición y tipo.

Las características de las Rugas Palatinas, a pesar del problema de descripción del patrón de la ruga palatina cuantitativa y cualitativamente, su singularidad para cada individuo ha sido reconocida en la ciencia forense como un potencial relevante de identificación debido a la estructura que lo componen. ${ }^{(9)}$ Existen numerosos sistemas de clasificación, algunos de ellos utilizados como base de todos los estudios descritos.

\section{Clasificación de las Rugas Palatinas}

Rugas Simples: recta (1), curva (2), angular (3), circular (4), ondulada (5), punto (6). Ver figura No. 1.

Rugas compuestas: se designaron con las abreviaturas Recta Curva(RC), Recta Curva Angular(RCA), Circular Recta(CR), Circular Sinusal Recta(CSR). ${ }^{(10)}$ Ver figura No. 2.
Figura $\mathrm{N}^{0} 1$. Tipos de rugas simples

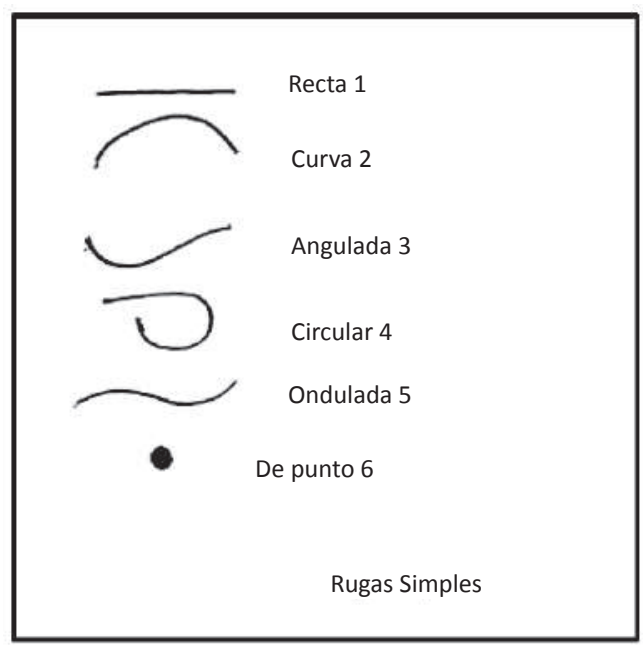

Figura No2 Tipos de rugas compuestas

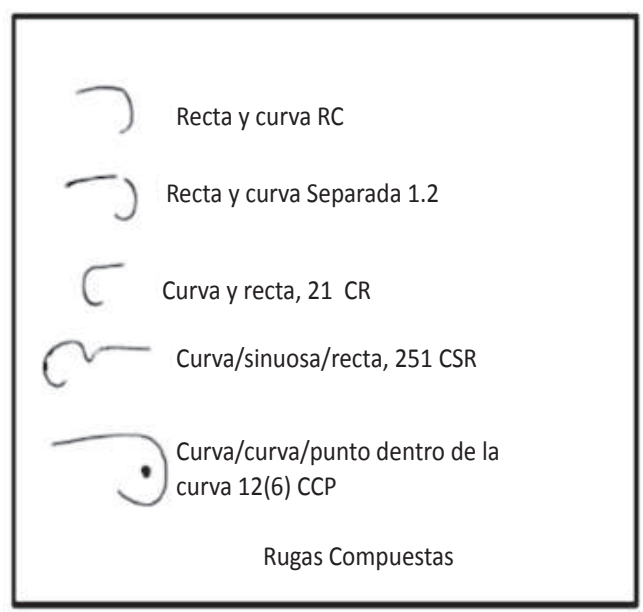

Fuente. Peñalver J. Odontología y DeontologíaOdontológica. Editorial Continente, Caracas, 1955

\section{PACIENTES Y METODOS:}

Estudio transversal descriptivo, realizado en las instalaciones clínicas de odontología en la UNAH-VS en el periodo intensivo 2012 con alumnos de último año de esta carrera.

Se tomó una muestra aleatoria de 20 personas, hombres y mujeres de diferentes edades, quienes firmaron un consentimiento informado previa explicación del estudio. 
Se realizó la toma de impresión del maxilar utilizando una cubeta de metal de tamaño adecuado,con alginato como material de impresión y el vaciado en yeso calcinado. Luego se procedió a la Calcorugoscopia, como se observa en la figura No 3 .

Figura № 3 Impresión Dental del estudio

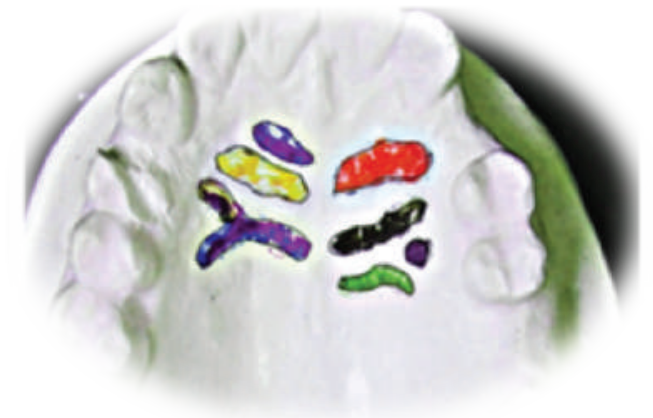

El duplicado obtenido se dividió por la línea media y se registraron las formas y números de rugosidades palatinas de cada lado.

El paso siguiente fue el llenado de ficha de cada uno de los modelos pertenecientes a los individuos. Se utilizó el programa Excel.

\section{RESULTADOS}

Mediante el análisis de la forma, la frecuencia en el estudio de ambas mitades de paladar además de la constancia de las rugas, señalamos los hallazgos obtenidos.

De los 20 pacientes estudiados la forma más común encontrada en el paladar derecho fue las curva (24 rugas), en el paladar izquierdo fue la recta (27 rugas). Ver tabla 1 y 2.

El mayor número de rugas palatinas en un individuo fue 12 y el menor número de rugas palatinas fue 5 . Ver Tabla No. 3.
Tabla $\mathbf{N}^{\circ}$ 1. Forma y número de rugas de cada individuo en la región derecha del paladar

\begin{tabular}{|c|c|c|c|c|c|c|c|c|c|c|}
\hline Individuo & Punto & Recta & Curva & Angulc & Circul & Sinuos & $\mathrm{RC}$ & & CR CS & $\begin{array}{l}\text { Total } \\
\text { Ruga: }\end{array}$ \\
\hline 1 & & 1 & 1 & 1 & & 1 & & & & 4 \\
\hline 2 & 2 & 2 & 1 & & & & 1 & & & 6 \\
\hline 3 & 1 & 2 & & & & & & & 1 & 4 \\
\hline 4 & 1 & 1 & 1 & & & 1 & & & & 4 \\
\hline 5 & & & 4 & & & & & & & 4 \\
\hline 6 & 2 & 1 & 2 & & & 2 & & & & 7 \\
\hline 7 & & 3 & 2 & & & & & & & 5 \\
\hline 8 & & & 1 & & & 1 & 1 & & & 3 \\
\hline 9 & & 2 & & & & & 1 & & & 3 \\
\hline 10 & & 1 & 2 & & & & & & & 3 \\
\hline 11 & & 1 & 1 & & & & & & & 2 \\
\hline 12 & & & 3 & & & & & & & 3 \\
\hline 13 & & 1 & & & & & 1 & & & 2 \\
\hline 14 & & 1 & 2 & & & & & & & 3 \\
\hline 15 & & & & & & 3 & 1 & & & 4 \\
\hline 16 & 1 & 1 & 1 & & & & 2 & & & 5 \\
\hline 17 & 1 & 2 & & & & & & & 1 & 4 \\
\hline 18 & & 2 & 2 & & & & & & & 4 \\
\hline 19 & & & & & & 1 & 1 & & & 2 \\
\hline 20 & & 1 & 1 & 1 & & & & & & 3 \\
\hline Total & 8 & 22 & 24 & 2 & 0 & 9 & 8 & 0 & 11 & \\
\hline
\end{tabular}

Tabla $\mathbf{N}^{\circ} 2$. Forma y número de rugas de cada individuo en la región izquierda del paladar

\begin{tabular}{|c|c|c|c|c|c|c|c|c|c|}
\hline Individuc & Recta & Curva & Angulos & & & Punto & RC RCA & CR CSR & $\begin{array}{l}\text { Total } \\
\text { Rugas }\end{array}$ \\
\hline 1 & 1 & 1 & & & 1 & & 1 & & 4 \\
\hline 2 & 3 & 1 & & & & & & 1 & 5 \\
\hline 3 & 1 & 3 & & & & 1 & & & 5 \\
\hline 4 & & 2 & & & & & 1 & & 3 \\
\hline 5 & 1 & 2 & & & 1 & & & & 4 \\
\hline 6 & 3 & 1 & & & 1 & & & & 5 \\
\hline 7 & 1 & & & & 1 & & 1 & & 3 \\
\hline 8 & 1 & & 2 & & 1 & & 1 & & 5 \\
\hline 9 & 2 & & & & 1 & 1 & & & 4 \\
\hline 10 & 2 & & & & 1 & 1 & & & 4 \\
\hline 11 & 2 & & & & & 1 & & & 3 \\
\hline 12 & 1 & & & & 1 & 1 & & & 3 \\
\hline 13 & 2 & & & & & & 11 & & 4 \\
\hline 14 & 1 & & 1 & & & & & & 2 \\
\hline 15 & & & & 1 & & & & 1 & 2 \\
\hline 16 & 2 & & & & 1 & 1 & 1 & & 5 \\
\hline 17 & 1 & 2 & 1 & & & 1 & & & 5 \\
\hline 18 & & & & & & 1 & 1 & & 2 \\
\hline 19 & 3 & 1 & & & & 1 & & & 5 \\
\hline 20 & & 2 & & & & 1 & & 1 & 4 \\
\hline Total & 27 & 15 & 4 & 1 & 9 & 10 & $\begin{array}{ll}7 & 1\end{array}$ & 30 & \\
\hline
\end{tabular}

Fuente: Instrumento de recolección de datos 
Tabla $\mathbf{N}^{\circ}$ 3: Número totales de rugas por cada individuo

\begin{tabular}{cc}
\hline Individuo & $\begin{array}{c}\text { Número } \\
\text { de Rugas }\end{array}$ \\
\hline 1 & 8 \\
\hline 2 & 11 \\
\hline 3 & 9 \\
\hline 4 & 7 \\
\hline 5 & 8 \\
\hline 6 & 12 \\
7 & 8 \\
8 & 8 \\
\hline 9 & 7 \\
\hline 10 & 7 \\
\hline 11 & 5 \\
\hline 12 & 6 \\
\hline 13 & 6 \\
\hline 14 & 5 \\
\hline 15 & 6 \\
\hline 16 & 10 \\
\hline 17 & 9 \\
\hline 18 & 6 \\
\hline 19 & 7 \\
\hline 20 & 7 \\
\hline
\end{tabular}

Fuente: Instrumento de recolección de datos

\section{DISCUSIÓN}

La cantidad y forma de rugas palatinas es diferente de un individuo a otro. Las rugas palatinas no son iguales en ambas mitades del paladar de un individuo y existen formas de rugas palatinas constantes en todas las personas. En un estudio del Dr. Luis Carter Merino de la Universidad de Chile sobre Palatoscopia y su aplicación en la identificación humana dice, "si comparamos los diversos sistemas actuales de identificación de que se ha ayudado el hombre para su diferenciación, veremos que ninguno es tan perfecto, de modo que, hay necesidad de recurrir al máximo de regiones útiles de nuestro organismo aprovechables a tal fin".

Basado en los estudios de los doctores Juan Ubaldo Carrea, Pedro A Trobo y otros, realizamos estudios y comprobamos que en el paladar poseemos una zona conocida como: Rugo Palatina de indiscutible importancia y de fácil práctica para que se le aproveche en la identificación individual, la cual posee ventajas en lo que se refiere a la parte débil del sistema Dactiloscópico, es decir, a lo expuesto a que se encuentra sometida la zona Digito-papilar que en los grandes accidentes resulta ser de frecuente destrucción. ${ }^{(6)}$

El método identificatorio a través de la Rugoscopia Forense puede considerarse como una técnica odontológica de identificación en carácter de proposición ya que es necesario uniformar las formas de clasificación y registro. ${ }^{(6)}$ No existe la presunción y se recomienda que al tomar impresiones de pacientes, cerciorarse que ambas mitades del paladar estén reproducidas correcta mente, ya que en un mismo paciente estas son diferentes y en caso de desastres masivos la mitad del paladar puede llevar a contribuir con la identificación del cadáver.

En conclusión la Rugoscopia es un método sencillo, de bajo costo y aplicable a distintos grupos poblacionales, creándose bases de datos y modelos pre-mortem que puede ser utilizados dado el caso, para ser comparado con registros post-mortem y lograr una identificación positiva, lo que implica un método de gran utilidad en la individualización de seres humanos dentro de la Odontología Forense. Se recomienda aconsejar a las autoridades pertinentes, colegio de cirujanos dentistas, odontólogos en general a tener como antecedente ante-morten el diseño de rugas palatinas en cada uno de los pacientes atendidos así como mostrar a Medicina Forense y al Registro Nacional de las personas la importancia de obtener y mantener una base de datos Rugoscopicos como método de identificación. 


\section{BIBLIOGRAFÍA}

1. Vargas E. Medicina Forense y Deontologíamédica: Ciencias forenses para médicos y abogados. Editorial Trillas; 1991.

2. Lubian y Arias R. Historia de la Dactiloscopia. España: Editorial Reus; 2010.

3. Foglia, P. Grafología Forense: Tendencias criminales en la escritura. Buenos Aires; 2003.

4. Aguilar, M. Presunción de Inocencia. Mexico: Editorial Azteca; 2006.

5. Joya Amendola B. Informe: Así me acusaron. Honduras; 2011.

6. Ciocca Gómez L. Identificación en desastres masivos. Santiago; 1992.

7. Fonseca, G y Rodríguez, F. Propuesta de Codificación y Análisis de Rugosidades Palatinas para su aplicación en
Odontología Antropológica y Forense Oral. Rev Oral; 2009; 31: 518 - 522. [citado el 2014 Julio 15]

Disponible en www.medigraphic.com/ pdfs/oral/ora-2009/ora0931d.pdf.

8. Ciocca Gómez Luis, OdontologíaMédico-Legal, Ediciones Jurídicas de Santiago, Septiembre 2009.

9. Negre Muñoz, María del Carmen. Nuevas aportaciones al procesado de huellas labiales: los lisocromos en queiloscopia. Valencia; Junio 2004. [citado 2014 Agosto 4]. Disponible en http:// www.tdx.cat/bitstream/handle/ 10803/10057/negre.pdf;jsessionid= 776CC2E7BE536144997340712B00F 198.tdx1?sequence=1.

10. Peñalver J. Odontología y Deontología Odontológica. Caracas: Editorial Continente; 1955. 